Pacific Journal of Mathematics

APPROXIMATION OF WIENER INTEGRALS OF
FUNCTIONALS CONTINUOUS IN THE UNIFORM TOPOLOGY 


\title{
APPROXIMATION OF WIENER INTEGRALS OF FUNCTIONALS CONTINUOUS IN THE UNIFORM TOPOLOGY
}

\author{
H. C. Finlayson
}

\begin{abstract}
The result obtained in this paper is a technique for the approximation and estimation of error of Wiener integrals of suitable functionals continuous in the uniform topology. For a certain class of functionals called third degree polynomials exact results occur at the first as well as each subsequent stage of approximation.
\end{abstract}

Similar results for functionals continuous in the Hilbert topology are given in [1], [4], [5], [6] and [7]. In each of these papers the functions $x(s)$ of Wiener space are approximated by linear combinations of the first $n$ indefinite integrals $\left\{\beta_{i}(t)\right\}$ of a certain complete set of orthonormal functions $\left\{\alpha_{i}(t)\right\}$. The approximation for $x(t)$ turns out to be $\sum_{i=1}^{n} c_{i}(x) \beta_{i}(t)$ where the $c_{i}(x)$ 's are Stieltjes integrals of $x(s)$ with respect to the $\alpha$ 's. When $x(t)$ is replaced by this approximation in $F[x(\cdot)]$ a standard Wiener integration formula can be applied. If $F$ is required to be continuous in the Hilbert topology, [4] and [5] show there is (as might be expected) considerable choice in the C.O.N. set. However the uniform topology seems more natural to use in Wiener space and when continuity in this topology is required it may be there is not so large a choice. The Haar functions seem a reasonable choice to try and it is these the author has used.

Let $C$ be the space of real functions continuous on $[0,1]$ and which vanish at zero. Let $\left\{h_{n}(s)\right\}$ be the Haar functions normalized to be right continuous and to vanish at $s=1$. The approximation of this paper applies to $F[x]$ if

$$
\begin{aligned}
F\left[x_{0}+x\right]= & F\left[x_{0}\right] \\
& +\sum_{i=1}^{3} \int_{0}^{1}(i) \int_{0}^{1} x\left(s_{1}\right) \cdots x\left(s_{i}\right) d_{(i)} K_{i}\left(x_{0} \mid s_{1}, \cdots, s_{i}\right)+Q\left[x_{0}, x\right]
\end{aligned}
$$

where, with $\|x\|=\max _{t}|x(t)|,\left|Q\left[x_{0}, x\right]\right| \leqq A\|x\|^{D} \exp B\left(\left\|x_{0}\right\|^{2}+\|x\|^{2}\right)$ with $B<1 / 12$ and $D>0$.

Notation. Let $\left\{h_{n}(s)\right\}$ be the Haar functions on $[0,1]$ normalized to be right continuous and so $h_{n}(1)=0$.

Let, for $n=1,2,3, \cdots$,

$$
c_{n}(x)=-\int_{0}^{1} x(s) d h_{n}(s),
$$




$$
\begin{aligned}
\beta_{n}(t) & =\int_{0}^{t} h_{n}(s) d s, \\
x^{n}(t) & =\sum_{i=1}^{n} c_{i}(x) \beta_{i}(t), \\
\psi_{n}(\xi, t) & =\sum_{i=1}^{n} \xi_{i} \beta_{i}(t), \\
e_{n}(\xi) & =(2 \pi)^{-n / 2} \exp \left[\left(-\xi_{1}^{2}-\cdots-\xi_{n}^{2}\right) / 2\right] .
\end{aligned}
$$

(This is the kernel commonly used now whereas that used in [1], [4] and [5] was $\pi^{-n / 2} \exp \left(-\xi_{1}^{2}-\cdots-\xi_{n}^{2}\right)$.)

Finally let

$$
\|x\|=\sup _{t \in[0,1]}|x(t)|
$$

for $x \in C$ and let

$$
\rho(s, t)=\left(2^{3 / 2} / \pi\right) \sum_{k=1}^{\infty} \sin \left(k-\frac{1}{2}\right) \pi t h_{k}(s) /(2 k-1)
$$

(that this last series converges for $(s, t) \in[0,1] \times[0,1]$ and is, for fixed $s$, continuous in $t$ will be seen in Theorem 1. Also $\rho(s, 0)=0$ and so, for fixed $s, \rho(s, t)$ is in $C$ and $\rho^{n}(s, t)$ can be computed).

In connection with Radon integrals the symbol $\int_{0}^{1}$ will be used rather than $\int_{0}^{1}(n) \int_{0}^{1}$ and $d$ subscripted with $n$ subscripted $s$ 's will be replaced by $d_{(n)}$. Another abbreviation is given by the following equation:

$$
\int_{-\infty}^{\infty} G(f(\xi), n) d \mu_{m}=\int_{-\infty}^{\infty}(n) \int_{-\infty}^{\infty} e_{n}(\xi) G(f(\xi), n) d \xi_{i} \cdots d \xi_{n} .
$$

If $F[x]$ is defined on $C$ we define $I_{n}(F)$ and $J_{n}(F)$ by the following equations provided the right hand sides have meaning.

$$
\begin{aligned}
I_{n}(F)= & \int_{-\infty}^{\infty} F\left[\psi_{n}(\xi, \cdot)\right] d \mu_{n}, \\
J_{n}(F)= & \frac{1}{2} \int_{-\infty}^{\infty} \int_{0}^{1}\left\{F\left[\psi_{n}(\xi, \cdot)+\rho(s, \cdot)-\rho^{n}(s, \cdot)\right]\right. \\
& \left.+F\left[\psi_{n}(\xi, \cdot)-\rho(s, \cdot)+\rho^{n}(s, \cdot)\right]\right\} d s d \mu_{n} .
\end{aligned}
$$

2. The principal theorem. The following theorem and corollary are the main results of the paper.

THeOREM 6. Let $F[x]$ be integrable on $C$ and such that $J_{n}(F)$ : $n=1,2,3, \cdots$ exists as a finite quantity. For each $x_{0} \in C$ let:

$K_{i}\left(x_{0} \mid s_{1}, \cdots, s_{i}\right), i=1,2,3$, be right continuous and of bounded variation in any $j(j \leqq i)$ of the variables for the other $i-j$ variables fixed. For each pair $\left[x_{0}, x\right] \in C \times C$ 


$$
\text { let } P\left[x_{0}, x\right]=F\left[x_{0}\right]+\sum_{i=1}^{3} \int_{0}^{1} x\left(s_{1}\right) \cdots x\left(s_{i}\right) d_{(i)} K\left(x_{0} \mid s_{1}, \cdots, s_{i}\right) .
$$

Let $F\left[x_{0}+x\right]=P\left[x_{0}, x\right]+Q\left[x_{0}, x\right]$ define $Q\left[x_{0}, x\right]$. Then if $\left|Q\left[x_{0}, x\right]\right| \leqq$ $A\|x\|^{D} \exp B\left[\left\|x_{0}\right\|^{2}+\|x\|^{2}\right]$, where $B<1 / 12$, and $D>0$ and if $\alpha \in(0$, 1/2) there follows

$$
\left|J_{n}(F)-\int_{0} F[x] d x\right|=o\left(n^{-\alpha D}\right) \text { as } n \rightarrow \infty .
$$

Furthermore, if $Q=0$ then

$$
J_{n}(F)=\int_{c} F[x] d x \text { for each } n .
$$

COROLlaRY. Under the conditions of the above theorem a specific estimate of error is given by

$$
\begin{aligned}
& \left|J_{n}(F)-\int_{c} F[x] d x\right| \\
& \quad \leqq A\left\{M^{2 / 3}\left[2^{\alpha+1} / n^{\alpha}\right]^{D}[2 / \sqrt{1-12 B}]^{1 / 3}\right. \\
& \quad+[2 / \sqrt{1-4 B}][31 / \sqrt{n}]^{D} \exp \left[31^{2} B / n\right]
\end{aligned}
$$

where $M$ is the constant given in Lemma 2 with $P$ replaced by $3 D / 2$.

The following theorems (except Theorem 2) and two lemmas are the main results used in the proof of Theorem 6. These theorems are analogous to correspondingly numbered theorems in [4]. In fact Theorems 3 and 5 are identical to those of [4] and so proofs for them will not be given.

THEOREM 1. (i) The $\rho(s, t)$ series converges, the convergence being uniform in $(s, t) \in[0,1] \times[0,1]$.

(ii) $\rho(s, t)$ is continuous in $t$ for each fixed $s$.

(iii) $\left\|\rho(s, \cdot)-\rho^{n}(s, \cdot)\right\|$ is measurable in $s$.

(iv) $\left\|\rho(s, \cdot)-\rho^{n}(s, \cdot)\right\| \leqq 31 / n^{1 / 2}$.

(v) If, for $x \in C, F[x]=K_{0}+\sum_{i=1}^{3} \int_{0}^{1} x\left(s_{1}\right) \cdots x\left(s_{i}\right) d_{(i)} K_{i}\left(s_{1}, \cdots, s_{i}\right)$ in which the $K_{i}$ 's are right continuous and of bounded variation in any $j(j \leqq i)$ of the variables for the other $i-j$ variables fixed then

$$
\int_{c} F[x] d x=\frac{1}{2} \int_{0}^{1}\{F[\rho(s, \cdot)]+F[-\rho(s, \cdot)]\} d s .
$$

(The reason $\sqrt{2}$ does not appear under the $\rho$ 's as in Theorem 1 of [4] is the change in kernel which results in $\int_{c} x(s) x(t) d x$ being $\frac{1}{2} \min (s, t)$ rather than $\min (s, t)$.) 
Lemma 1. (Ciesielski [2]). For each $x \in C$, the graph of $x^{n}(t)$ is an inscribed polygon of the graph of $x(t)$. The graph of $x^{n+1}(t)$ has at least the same vertices as that of $x^{n}(t)$ and $\left\{x^{n}(t)\right\}$ converges uniformly to $x(t)$.

Some notation, now to be given, is used in Lemma 2 below. For fixed $x \in C$ and $\alpha \in\left(0, \frac{1}{2}\right)$ let $\varphi_{\alpha}[x]$ be the infimum of $h>0$ such that $\left|x\left(t^{\prime}\right)-x\left(t^{\prime \prime}\right)\right| \leqq h\left|t^{\prime}-t^{\prime \prime}\right|^{\alpha}$ for $t^{\prime}$ and $t^{\prime \prime}$ in $[0,1]$. (that such $h$ exists for almost all $x \in C$ has been shown by $\mathrm{N}$. Wiener [10]).

Lemma 2. (Yeh [8]). For every $\alpha \in(0,1 / 2)$ and $P>0$, the functional $\left\{\varphi_{\alpha}[x]\right\}^{P}$ is Wiener integrable i.e., $\int_{c}\left\{\varphi_{\alpha}[x]\right\}^{P} d x<\infty$.

In fact for any $N>\frac{1}{2} \max \{(1+2 \alpha) /(1-2 \alpha), P\}$,

$$
\int_{c}\left\{\varphi_{\alpha}[x]\right\}^{P} d x \leqq M
$$

where

$$
M=(2 N)^{N} e^{-N}\left\{1-2^{1 / 2+\alpha-N(1-2 \alpha)}\right\}^{-1} \sum_{m=1}^{\infty}(m+1)^{P} /(2 N+1)<\infty .
$$

THEOREM 2. If $F[x]$ is continuous in the uniform topology on $C$ and if either

(i) $F[x]$ is bounded

or

(ii) there exist nondecreasing $G_{1}(u)$ and $G_{2}(u)$ defined on $[0, \infty)$ such that $G_{1}\left[\max _{t \in[0,1]} x(t)\right]$ and $G_{2}\left[\max _{t \in\{0,1\}}\{-x(t)\}\right]$ are Wiener integrable and such that

$$
\mid F[x] \leqq G_{1}\left[\max _{t \in[0,1]} x(t)\right]+G_{2}\left[\max _{t \in[0,1]}\{-x(t)\}\right]
$$

then

$$
\lim _{n \rightarrow \infty} I_{n}(F)=\int_{c} F[x] d x .
$$

Particular suitable choices for $G_{1}$ and $G_{2}$ are

$$
G_{1}(u)=G_{2}(u)=M \exp \left\{h u^{p}\right\} \text { for } p \in[0,2) \text { and arbitrary real }
$$
$M$ and $h$.

(2.4) $G_{1}(u)=G_{2}(u)=M \exp \left\{h u^{2}\right\}$ for $h<\frac{1}{2}$ and arbitrary real $M$.

TheOREM 3. If $F[x] \in L_{1}(C)$ then

$$
\int_{c} F[x] d x=\int_{-\infty}^{\infty} \int_{c} F\left[x(\cdot)-x^{n}(\cdot)+\psi_{n}(\xi, \cdot)\right] d x d \mu_{n} .
$$


Theorem 4. For $\alpha \in(0,1 / 2)$ and $P \geqq 0$,

$$
\int_{c}\left\|x-x^{n}\right\|^{P} d x \leqq M\left[2^{\alpha+1} / n^{\alpha}\right]^{P}
$$

where $M$ is as in Lemma 2.

THEOREM 5. For fixed $i \in\{1,2,3\}$, let $H\left(t_{1}, \cdots, t_{i}\right)$ be right continuous and of bounded variation in any $j(j \leqq i)$ of its variables for the other $i-j$ variables fixed. Then there exists $N\left(s_{1}, \cdots, s_{i}\right)$ of bounded variation and right continuous such that for all $x \in C$,

$$
\int_{0}^{1}\left[x\left(t_{1}\right)-x^{n}\left(t_{1}\right)\right] \cdots\left[x\left(t_{i}\right)-x^{n}\left(t_{i}\right)\right] d_{(i)} H\left(t_{1}, \cdots, t_{i}\right)
$$

is of the form

$$
\int_{0}^{1} x\left(s_{1}\right) \cdots x\left(s_{i}\right) d_{(i)} N\left(s_{1}, \cdots, s_{i}\right) .
$$

The proof of Theorem 6 and its corollary follows. Let

$$
\begin{aligned}
\varepsilon_{n}= & \int_{c} F[x] d x-J_{n}(F) \\
= & \int_{c} F[x] d x \\
& -\int_{-\infty}^{\infty} \frac{1}{2} \int_{0}^{1}\left\{F\left[\psi_{n}(\xi, \cdot)+\rho(s, \cdot)-\rho^{n}(s, \cdot)\right]\right. \\
& \left.+F\left[\psi_{n}(\xi, \cdot)-\rho(s, \cdot)+\rho^{n}(s, \cdot)\right]\right\} d s d \mu_{n} .
\end{aligned}
$$

If now $F$ is replaced by $P+Q$ the integrals can be combined and, because of Theorems 1,3 and 5 , the part involving $P$ disappears. (The detailed argument is exactly the same as that in [4, pp. 64-65] where all symbols and theorems used there are to be replaced by the corresponding ones of this paper. See also the note after (2.1)). What is left is

$$
\begin{aligned}
\varepsilon_{n}= & \int_{-\infty}^{\infty}\left\{\int_{c} Q\left[\psi_{n}(\xi, \cdot), x(\cdot)-x^{n}(\cdot)\right] d x\right. \\
& -\frac{1}{2} \int_{0}^{1}\left(Q\left[\psi_{n}(\xi, \cdot), \rho(s, \cdot)-\rho^{n}(s, \cdot)\right]\right. \\
& \left.+Q\left[\psi_{n}(\xi, \cdot),-\rho(s, \cdot)+\rho^{n}(s, \cdot)\right] d s\right\} d \mu_{n} .
\end{aligned}
$$

If

$$
\left|Q\left[x_{0}, x\right]\right| \leqq A\|x\|^{D} \exp B\left[\left\|x_{0}\right\|^{2}+\|x\|^{2}\right]
$$

then 


$$
\begin{aligned}
\left|\varepsilon_{n}\right| \leqq & A \int_{-\infty}^{\infty}\left\{\int_{c}\left\|x-x^{n}\right\|^{D} \exp B\left[\left\|\psi_{n}(\xi, \cdot)\right\|^{2}+\left\|x-x^{n}\right\|^{2}\right] d x\right. \\
& +\int_{0}^{1}\left\|\rho(s, \cdot)-\rho^{n}(s, \cdot)\right\|^{D} \exp B\left[\left\|\psi_{n}(\xi, \cdot)\right\|^{2}\right. \\
& \left.\left.+\left\|\rho(s, \cdot)-\rho^{n}(s, \cdot)\right\|^{2}\right] d s\right\} d \mu_{n} \cdot
\end{aligned}
$$

Now steps almost identical to those of $\left[4\right.$, pp. 66-67] with $\|x\|^{2}$ replacing $\int_{0}^{1}[x(s)]^{2} d s$ and $a^{2}+b^{2}=\left[(a+b)^{2}+(a-b)^{2}\right] / 2$ replaced by

$$
\begin{gathered}
\|x\|^{2}+\|y\|^{2} \leqq\|x+y\|^{2}+\|x-y\|^{2} \text { yield } \\
\int_{-\infty}^{\infty}\left\{\int_{c}\left\|x-x^{n}\right\|^{D} \exp B\left[\left\|\psi_{n}(\xi, \cdot)\right\|^{2}+\left\|x-x^{n}\right\|^{2}\right] d x\right\} d \mu_{n} \\
\leqq\left[\int_{c}\left\|x-x^{n}\right\|^{3 D / 2} d x\right]^{2 / 3}\left[\int_{c} \exp 6 B\|x\|^{2} d x\right]^{1 / 3}
\end{gathered}
$$

and

$$
\begin{aligned}
& \int_{-\infty}^{\infty} \exp B\left\|\psi_{n}(\xi, \cdot)\right\|^{2} d \mu_{n} \int_{0}^{1}\left\|\rho(s, \cdot)-\rho^{n}(s, \cdot)\right\|^{D} \\
& \quad \exp B\left\|\rho(s, \cdot)-\rho^{n}(s, \cdot)\right\|^{2} d s \leqq \int_{c} \exp 2 B\|x\|^{2} d x . \\
& \quad \int_{0}^{1}\left\|\rho(s, \cdot)-\rho^{n}(s, \cdot)\right\|^{D} \exp \left[B\left\|\rho(s, \cdot)-\rho^{n}(s, \cdot)\right\|^{2}\right] d s .
\end{aligned}
$$

Finally one notes that

so that for $K \in(0,1)$

$$
\|x\|=\max \left\{\max _{t \in[0,1]} x(t), \max _{t \in[0,1]}[-x(t)]\right\}
$$

$$
\exp \left(K\|x\|^{2}\right) \leqq \exp \left(K\{\max x(t)\}^{2}\right)+\exp \left(K\{\max [-x(t)]\}^{2}\right)
$$

and

$$
\begin{aligned}
& \int_{c} \exp \left(K\|x\|^{2}\right) d x \leqq 2 \int_{c} \exp \left(K\{\max x(t)\}^{2}\right) d x \\
& \quad=4 \int_{0}^{\infty} \exp \left[-(1-2 K) u^{2} / 2\right] d u / \sqrt{(2 \pi)}=2 / \sqrt{1-2 K} .
\end{aligned}
$$

(for the distribution of $\max x(t)$ see [3]).

The estimate (2.7) used first with Theorem 4 and then with Theorem 1 (iv) provides the estimates of the right sides of (2.5) and (2.6). The estimate given in the corollary follows at once as does also the order estimate of the theorem.

3. Proof of Theorems 1,2 and 4. As noted in $\S 2$ after the statement of Theorem 6, only Theorems 1, 2, and 4 remain to be 
proved. Yeh's lemma [8] and Ciesielski's lemma [2] provide a proof for Theorem 4. The lemma due to Ciesielski will be used in the proofs of Theorems 1 and 2. An outline of the proof of this lemma will follow. First there will be noted that there is a natural double indexing of the Haar functions:

$$
\begin{aligned}
& \alpha_{0}^{(0)}(s), \\
& \alpha_{n}^{(k)}(s): n=0,1,2, \cdots, k=1,2, \cdots, 2^{n} .
\end{aligned}
$$

A corresponding double indexing applies to the $\beta$ 's. It will be convenient to speak of "the $n$th cycle of $\alpha$ 's $\left(\beta^{\prime} \mathrm{s}\right): n \geqq 0$ " by which will be meant $\left\{\alpha_{n}^{(k)}: k=1,2, \cdots, 2^{n}\right\}$ (or similar for $\beta$ 's). Note that $\alpha_{0}^{(0)}$ is not in a cycle. Now it is fairly easy to prove by induction than any partial sum of the $c \beta$-series to at least the end of the $(N-1)^{\text {th }}$ cycle gives the value of $x(t)$ for all $t$ of the form $l / 2^{N}: l=1,2, \cdots, 2^{N}$ and that the graph of this partial sum is polygonal with vertices precisely those points where the graph of the partial sum agrees with the graph of $x(t)$. The conclusions of the lemma are thus obtained.

The proof of Theorem 2 follows:

First there is noted that a functional continuous in the uniform topology is Wiener measurable. Lemma 1 together with Lebesgue's bounded or dominated convergence theorem completes the proof of (i) or (ii) respectively. That (2.3) or (2.4) provide suitable choices for the $G$ 's follows from the formula for the integral of a functional of $\max x(t)$ which yields

$$
\begin{aligned}
\int_{c} G_{1}[\max x(t)] d x & =\int_{c} G_{1}[\max -\{x(t)\}] d x \\
& =2 \int_{0}^{\infty} e^{-\xi^{2} / 2} G_{1}(\xi) d \xi / \sqrt{(2 \pi)} \\
& =2 A \int_{0}^{\infty} e^{-\xi^{2} / 2+h \xi^{p}} d \xi / \sqrt{(2 \pi)}
\end{aligned}
$$

and the last integral clearly converges for the conditions given on $p$ and $h$ in (2.3) and (2.4).

Next is given the proof of Theorem 1 .

(i) For any fixed $s$ there is a most one $h_{k}(s)$ in "the $n^{\text {th }}$ cycle of Haar Functions" (for this notion c.f. beginning of outline of proof of the lemma) which is not zero and $\left|h_{k}(s)\right| \leqq \sqrt{2^{n}}$. But the $k$ for that $h_{k}(s)$ satisfies $k \geqq 1+1+2+4+\cdots+2^{n-1}=2^{n}$. A comparison of the series, after terms of value zero have been deleted, of

$$
\sum_{k=1}^{\infty}\left|\sin \left(k-\frac{1}{2}\right) \pi t h_{k}(s) /(2 k-1)\right|
$$


with the series

$$
\sum_{n=1}^{\infty}\left(\sqrt{2^{n}} / 2^{n}\right)
$$

which converges, yields the conclusion of (i).

(ii) That $\rho(s, t)$ is continuous in $t$ for each fixed $s$ follows at once from uniform convergence of a series of continuous functions.

(iii) To show that $\left\|\rho(s, \cdot)-\rho^{n}(s, \cdot)\right\|$ is measurable in $s, \rho^{n}(s, t)$ will first be calculated.

$$
\rho^{n}(s, t)=-\sum_{i=1}^{n} \int_{0}^{1} \rho(s, u) d h_{i}(u) \beta_{i}(t)
$$

(the Stieltjes integrals exist since $\rho(s, u)$ is continuous in $u$ )

$$
\begin{aligned}
& =\left(-2^{3 / 2} / \pi\right) \sum_{i=1}^{n} \int_{0}^{1}\left[\sum_{k=1}^{\infty} \sin \left(k-\frac{1}{2}\right) \pi u h_{k}(s) /(2 k-1)\right] d h_{i}(u) \beta_{i}(t) \\
& =\left(-2^{3 / 2} / \pi\right) \sum_{i=1}^{n} \sum_{k=1}^{\infty} \int_{0}^{1} \sin \left(k-\frac{1}{2}\right) \pi u d h_{i}(u) h_{k}(s) \beta_{i}(t) /(2 k-1)
\end{aligned}
$$

(because of uniform convergence of a series of continuous functions). Thus $\rho^{n}(s, t)$ is measurable in $s$ for each fixed $t$ and so of course is $\rho(s, t)$. Since $\rho^{n}(s, t)-\rho(s, t)$ is continuous in $t,\left\|\rho^{n}(s, \cdot)-\rho(s, \cdot)\right\|$ is determined by a countable number of $t$ values and so is measurable in $s$.

(iv) That

$$
\left\|\rho(s, \cdot)-\rho^{n}(s, \cdot)\right\| \leqq 31 / n^{1 / 2}
$$

uniformly in $s$ is seen as follows. Let $k$ be such that

$$
\begin{aligned}
& 1+1+2+4 \cdots+2^{n-1}\left(=2^{n}\right) \leqq k \\
& \leqq 1+1+2+4+\cdots+2^{n}\left(=2^{n+1}\right) .
\end{aligned}
$$

Note that $n \leqq \log _{2} k \leqq n+1$.

Now

$$
\begin{aligned}
\left|\rho(s, t)-\rho^{k}(s, t)\right|= & \left(2^{3 / 2} / \pi\right) \mid \sum_{i=1}^{\infty}\left[\sin \left(i-\frac{1}{2}\right) \pi t\right. \\
& \left.+\sum_{j=1}^{k} \int_{0}^{1} \sin \left(i-\frac{1}{2}\right) \pi u d h_{j}(u) \beta_{j}(t)\right] h_{i}(s) /(2 i-1) \mid .
\end{aligned}
$$

Since $\sin \left(i-\frac{1}{2}\right) \pi t \in C$, there follows from Lemma 1 that

$$
\left\|\sum_{j=1}^{k} \int_{0}^{1} \sin \left(i-\frac{1}{2}\right) \pi u d h_{j}(u) \beta_{j}(\cdot)\right\| \leqq\left\|\sin \left(i-\frac{1}{2}\right) \pi \cdot\right\|=1
$$

for all $i$. Now let the series (in $i$ ) for $\rho(s, t)-\rho^{k}(s, t)$ be split into two parts, viz. a finite sum from $i=1$ to $i=k$ and the remainder of the series from $i=k+1$ onward. The second of these two parts 
is estimated as follows:

$$
\left(2^{3 / 2} / \pi\right)\left|\sum_{i=k+1}^{\infty} \cdots\right| \leqq 2^{5 / 2} \sum_{i=n}^{\infty}\left(1 / \sqrt{2)^{i}} / \pi\right.
$$

(because of (3.1), the comparison series mentioned in the proof of Theorem 1(i), and the relation between $k$ and $n$ )

$$
=2^{5 / 2}\left(1 / \sqrt{2)^{n}} /[\pi(1-1 / \sqrt{2})] \leqq 32 /\left(\pi k^{1 / 2}\right) .\right.
$$

To estimate the first part it will be noted that, for any $i$, the maximum difference between the graph of $\sin \left(i-\frac{1}{2}\right) \pi t$ and the $k^{\text {th }}$ polygonal approximation, viz. $\left(\sin \left(i-\frac{1}{2}\right) \pi t\right)^{k}$, is no greater than the maximum slope of this sine curve multiplied by $1 / 2^{n}$. Thus

$$
\left\|\sin \left(i-\frac{1}{2}\right) \pi \cdot+\sum_{j=1}^{k} \int_{0}^{1} \sin \left(i-\frac{1}{2}\right) \pi u d h_{j}(u) \beta_{j}(\cdot)\right\| \leqq\left(i-\frac{1}{2}\right) \pi / 2^{n} \leqq i \pi / 2^{n}
$$

and therefore

$$
\left(2^{3 / 2} / \pi\right)\left|\sum_{i=1}^{k} \cdots\right| \leqq\left(2^{3 / 2} / \pi\right)\left[\pi / 2^{n}+\sum_{j=0}^{n}\left(2^{j+1} \pi / 2^{n}\right)(1 / \sqrt{2})^{j}\right]
$$

(because, for given $s$, the one function in the $j^{\text {th }}$ cycle of Haar functions which is not zero has index no greater than $2^{j+1}$ : the $\pi / 2^{n}$ before the summation is due to $\alpha_{0}^{(0)}$ which is not in a cycle)

$$
=\left(2^{3 / 2} / 2^{n}\right)\left[1+2 \sum_{j=0}^{n} \sqrt{2^{j}}\right] \leqq 20 / k^{1 / 2},
$$

and addition of the estimates completes the proof.

To prove (v) there is noted that the Fubini theorem for mixed Stieltjes and Wiener integrals will yield the required result if (2.1) can be shown to hold for $F[x]$ any one of the forms $K_{0}, x\left(s_{1}\right), x\left(s_{1}\right) x\left(s_{2}\right)$ and $x\left(s_{1}\right) x\left(s_{2}\right) x\left(s_{3}\right)$. But (2.1) clearly does hold for $K_{0}$ (yielding $K_{0}$ ) and for $x\left(s_{1}\right)$ and $x\left(s_{1}\right) x\left(s_{2}\right) x\left(s_{3}\right)$ (yielding 0 ). That (2.1) holds for $x\left(s_{1}\right) x\left(s_{2}\right)$ is seen from the computation

$$
\begin{aligned}
& \int_{0}^{1} \rho\left(u, s_{1}\right) \rho\left(u, s_{2}\right) d u \\
& \quad=\left(2^{3} / \pi^{2}\right) \sum_{k=1}^{\infty} \sin \left(k-\frac{1}{2}\right) \pi s_{1} \sin \left(k-\frac{1}{2}\right) \pi s_{2} /(2 k-1)^{2}=\min \left(s_{1}, s_{2}\right)
\end{aligned}
$$

(by Mercer's theorem for the integral equation

$$
\phi_{n}(s)=\lambda_{n} \int_{0}^{1} \min (s, t) \phi_{n}(t) d t .
$$

[9, p. 136] or [7, p. 464]) and the proof is complete.

Finally there follows the proof of Theorem 4 . Let $k$ be such that 


$$
\begin{array}{r}
1+1+2+4+\cdots+2^{n-1}\left(=2^{n}\right) \leqq k \\
\leqq 1+1+2+4+\cdots+2^{n}\left(=2^{n+1}\right)
\end{array}
$$

and let $t \in[0,1]$ be such that

$$
r / 2^{n} \leqq t \leqq(r+1) 2^{n}: 0 \leqq r \leqq 2^{n}-1 .
$$

Now (see notation in Yeh's lemma) for almost all $x \in C$

$$
\begin{gathered}
\left|x(t)-x\left(r / 2^{n}\right)\right| \leqq \phi_{\alpha}[x] / 2^{\alpha n} . \quad \text { Also } \\
\left|x^{k}\left(r / 2^{n}\right)-x^{k}(t)\right| \leqq\left|x^{k}\left(r / 2^{n}\right)-x^{k}\left([r+1] / 2^{n}\right)\right|
\end{gathered}
$$

(because the graph of $x^{k}$ is a chord of the graph of $x$ on $\left[r / 2^{n},(r+1) / 2^{n}\right]$ according to the Ciesielski lemma).

$$
=\left|x\left(r / 2^{n}\right)-x\left([r+1] / 2^{n}\right)\right|
$$

(since, from the Ciesielski lemma, $x$ and $x^{k}$ agree at $r / 2^{n}$ and $[r+1] / 2^{n}$ ),

$$
\leqq \phi_{\alpha}[x] / 2^{\alpha n}
$$

for almost all $x$.

Thus

$$
\begin{aligned}
\mid x(t)- & x^{k}(t) \mid \\
& =\left|x(t)-x\left(r / 2^{n}\right)+x\left(r / 2^{n}\right)-x^{k}(t)\right| \\
& =\left|x(t)-x\left(r / 2^{n}\right)+x^{k}\left(r / 2^{n}\right)-x^{k}(t)\right| \\
& \leqq 2 \phi_{\alpha}[x] / 2^{\alpha n}
\end{aligned}
$$

(because of the Schwarz inequality and inequalities (3.2) and (3.3)). From the fact that $n \geqq \log _{2} k-1$ there then follows for almost all $x$

$$
\left\|x-x^{k}\right\| \leqq 2^{\alpha+1} \phi_{\alpha}[x] / k^{\alpha}
$$

and an application of Yeh's lemma completes the proof.

\section{REFERENCES}

1. R.H. Cameron, A “Simpson's Rule" for the numerical evaluation of Wiener's integrals in function space, Duke Math. J. 18 (1951), 111-130.

2. Z. Ciesielski, On Haar functions and on the Schauder basis of the space $C_{\langle 0,1\rangle}$, (Russian summary, unbound insert), Bull. Acad. Polon. Sci. Sér. Sci. Math. Astronom. Phys. 7 (1959), 227-232.

3. P. Erdos \& M. Kac, On certain limit theorems of the theory of probability, Bull. Amer. Math. Soc. 52 (1946), 292-302.

4. H. C. Finlayson, New approximations for Wiener integrals, with error estimates, Canad. J. Math. 19 (1967), 58-105.

5. - Vladimirov-type approximation of Wiener integrals of suitably dominated functionals, Trans. Amer. Math. Soc. 132 (1968), 461-469. 
6. Alan G. Konheim and Willard L. Miranker, Mathematics of computation, 21 (1967), 49-65.

7. V.S. Vladimirov, The approximate evaluation of Wiener integrals, Uspehi Mat. Nauk 15 (1960), 129-135; English transl. Amer. Math. Soc. Trans. (2) 34 (1963), 405-412.

8. J. Yeh, Minimal coefficients in Hölder conditions in the Wiener space. (submitted for publication).

9. K. Yosheda, Lectures on differential and integral equations, New York, 1960.

10. N. Wiener, Generalized harmonic analysis, Acta Mathematica 55 (1930), 117-258.

Received September 25, 1969.

UNIVERSITY of MaNitoba 



\section{PACIFIC JOURNAL OF MATHEMATICS}

EDITORS

H. SAMELSON

Stanford University

Stanford, California 94305

RichaRd PIERCE

University of Washington

Seattle, Washington 98105
J. DUGUNDJI

Department of Mathematics

University of Southern California

Los Angeles, California 90007

RICHARD ARENS

University of California

Los Angeles, California 90024

\section{ASSOCIATE EDITORS}

E. F. BECKENBACH

B. H. NeumanN

F. WOLF

K. YOSHIDA

\section{SUPPORTING INSTITUTIONS}

UNIVERSITY OF BRITISH COLUMBIA

CALIFORNIA INSTITUTE OF TECHNOLOGY

UNIVERSITY OF CALIFORNIA

MONTANA STATE UNIVERSITY

UNIVERSITY OF NEVADA

NEW MEXICO STATE UNIVERSITY

OREGON STATE UNIVERSITY

UNIVERSITY OF OREGON

OSAKA UNIVERSITY

UNIVERSITY OF SOUTHERN CALIFORNIA
STANFORD UNIVERSITY

UNIVERSITY OF TOKYO

UNIVERSITY OF UTAH

WASHINGTON STATE UNIVERSITY

UNIVERSITY OF WASHINGTON

AMERICAN MATHEMATICAL SOCIETY CHEVRON RESEARCH CORPORATION TRW SYSTEMS

NAVAL WEAPONS CENTER 


\section{Pacific Journal of Mathematics}

May, 1970

Johan Aarnes, Edward George Effros and Ole A. Nielsen, Locally compact

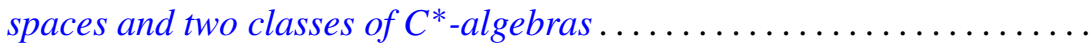

Allan C. Cochran, R. Keown and C. R. Williams, On a class of topological

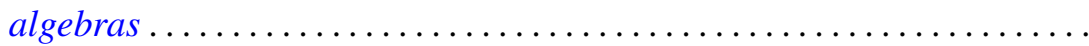

John Dauns, Integral domains that are not embeddable in division rings ....

Robert Jay Daverman, On the number of nonpiercing points in certain

crumpled cubes.....................................

Bryce L. Elkins, Characterization of separable ideals ................

Zbigniew Fiedorowicz, A comparison of two naturally arising uniformities

on a class of pseudo-PM spaces ...........................

Henry Charles Finlayson, Approximation of Wiener integrals of functionals

continuous in the uniform topology ........................

Theodore William Gamelin, Localization of the corona problem ...........

Alfred Gray and Paul Stephen Green, Sphere transitive structures and the

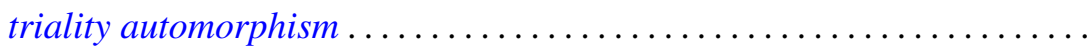

Charles Lemuel Hagopian, On generalized forms of aposyndesis ..........

J. Jakubík, On subgroups of a pseudo lattice ordered group ...............

Cornelius W. Onneweer, On uniform convergence for Walsh-Fourier

series..................................

Stanley Joel Osher, On certain Toeplitz operators in two variables ...

Washek (Vaclav) Frantisek Pfeffer and John Benson Wilbur, On the

measurability of Perron integrable functions............

Frank J. Polansky, On the conformal mapping of variable regions...

Kouei Sekigawa and Shûkichi Tanno, Sufficient conditions for a Riemannian manifold to be locally symmetric ...................

James Wilson Stepp, Locally compact Clifford semigroups ....

Ernest Lester Stitzinger, Frattini subalgebras of a class of solvable Lie

algebras ................................

George Szeto, The group character and split group algebras...

Mark Lawrence Teply, Homological dimension and splitting torsion

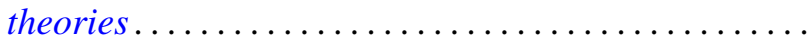

David Bertram Wales, Finite linear groups of degree seven. II ...

Robert Breckenridge Warfield, Jr., An isomorphic refinement theorem for

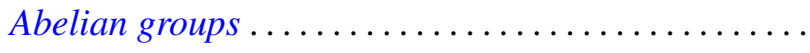

James Edward West, The ambient homeomorphy of an incomplete subspace

of infinite-dimensional Hilbert spaces................

Peter Wilker, Adjoint product and hom functors in general topology ...

Daniel Eliot Wulbert, A note on the characterization of conditional 